\title{
How Close Are We to Understanding What (if Anything) $\gamma$ Oscillations Do in Cortical Circuits?
}

\author{
(DVikaas S. Sohal \\ Department of Psychiatry, Weil Institute for Neurosciences, Kavli Center for Fundamental Neuroscience, and Sloan Swartz Center for Theoretical \\ Neurobiology, University of California, San Francisco, San Francisco, California 94143-0444
}

$\gamma$ oscillations, which can be identified by rhythmic electrical signals $\sim 30-100 \mathrm{~Hz}$, consist of interactions between excitatory and inhibitory neurons that result in rhythmic inhibition capable of entraining firing within local cortical circuits. Many possible mechanisms have been described through which $\gamma$ oscillations could act on cortical circuits to modulate their responses to input, alter their patterns of activity, and/or enhance the efficacy of their outputs onto downstream targets. Recently, several studies have observed changes in behavior after optogenetically manipulating neocortical $\gamma$ oscillations. Now, future studies should determine whether these manipulations elicit physiological correlates associated with specific mechanisms through which $\gamma$ oscillations are hypothesized to modulate cortical circuit function. There are numerous such mechanisms, so identifying which ones are actually engaged by optogenetic manipulations known to affect behavior would help flesh out exactly how $\gamma$ oscillations contribute to cortical circuit function under normal and/or pathological conditions.

\begin{abstract}
Introduction
$\gamma$ oscillations are rhythmic fluctuations of electrical activity (Buzsáki, 2005) that reflect underlying neuronal synchronization. The precise frequency band that corresponds to $\gamma$ oscillations is largely a matter of opinion, but the lower and upper limits are usually $20-30 \mathrm{~Hz}$ and $80-120 \mathrm{~Hz}$, respectively. $\gamma$ oscillations can be identified by electrical recordings on many scales including outside the skull (EEG), on the brain surface (ECoG), extracellular recordings within the brain (LFP, multinunit or single-unit activity), and subthreshold fluctuations within intracellular recordings. Critically, these electrical signals (e.g., increases in 30$100 \mathrm{~Hz}$ LFP power) are merely signatures of $\gamma$ oscillations, and on their own have no functional significance. Rather, the actual " $\gamma$ oscillation" comprises the synchronized rhythmic patterns of spiking and synaptic inhibition (described below) that give rise to these readily identified electrical signatures. (For the purposes of this review, we are not concerned with higher frequency " $\gamma$ oscillations" $>120 \mathrm{~Hz}$, which may reflect extracellular electrical consequences of action potential generation).
\end{abstract}

\section{Mechanisms of $\gamma$ oscillations}

It is well established that $\gamma$ oscillations reflect rhythmic firing of inhibitory interneurons, particularly (though not necessarily ex-

Received March 25, 2016; revised Aug. 8, 2016; accepted Aug. 9, 2016.

This work was supported by National Institutes of Health Grant 5R01MH106507. I thank Loren Frank, Andrea Hasenstaub, and Kathleen Cho for helpful comments on the manuscript; and Jess Cardin for discussions about this Dual Perspectives feature.

The authors declare no competing financial interests.

Correspondence should be addressed to Dr. Vikaas S. Sohal, University of California, San Francisco, 675 Nelson Rising Lane, San Francisco, CA 94143-044. E-mail: vikaas.sohal@ucsf.edu.

DOI:10.1523/JNEUROSCI.0990-16.2016

Copyright $\odot 2016$ the authors $\quad 0270-6474 / 16 / 3610489-07 \$ 15.00 / 0$ clusively) parvalbumin (PV)-expressing fast-spiking interneurons (Whittington et al., 1995; Csicsvari et al., 2003; Hasenstaub et al., 2005; Bartos et al., 2007; Cardin et al., 2009; Sohal et al., 2009; Buzsáki and Wang, 2012). Rhythmic interneuron firing drives $\gamma$-frequency fluctuations in synaptic inhibition, which are the principle sources of the electrical signatures (e.g., in LFPs) used to identify $\gamma$ oscillations. Rhythmic inhibition can entrain pyramidal neuron firing (Csicsvari et al., 2003; Hasenstaub et al., 2005; Carr et al., 2012; Kim et al., 2016). In many cases, $\gamma$ oscillations reflect interactions between excitatory neurons and inhibitory interneurons in which excitatory neurons fire, triggering the synchronized discharge of many inhibitory interneurons, which deliver feedback inhibition to excitatory neurons, transiently silencing them. When this inhibition wears off, excitatory neurons fire again, triggering a new cycle of the $\gamma$ oscillation. In other cases, interactions between inhibitory neurons themselves may suffice to generate $\gamma$ oscillations, and the relative contributions of these two mechanisms to various forms of $\gamma$ oscillations remain unclear (Tiesinga and Sejnowski, 2009). Regardless, in both cases, the " $\gamma$ oscillation" constitutes rhythmic synaptic inhibition that regulates the firing of many neurons in a local circuit.

\section{$\gamma$ oscillations and behavior}

$\gamma$ oscillations can be observed throughout the cerebral cortex and under a wide range of conditions, which usually correspond to the cortex being "active." That is, in sensory cortex, they are typically observed in the presence of sensory stimulation (Gray and Singer, 1989; Gregoriou et al., 2009; Siegle et al., 2014). Highorder regions of associational cortex typically exhibit $\gamma$ oscillations when engaged in cognitive tasks, e.g., $\gamma$ oscillations in the hippocampus are linked to working memory (Yamamoto et al., 2014) and replay (Carr et al., 2012), and prefrontal $\gamma$ oscillations 
increase during tasks that involve attention (Kim et al., 2016), cognitive control (Cho et al., 2015), and social interaction (Cho et al., 2015). In the hippocampus, the power spectrum of LFP recordings exhibits a discrete "bump" at either $30-50 \mathrm{~Hz}$ ("slow $\gamma$ ") or $60-100 \mathrm{~Hz}$ ("fast $\gamma$ ") (Colgin et al., 2009). In visual cortex, visual stimuli, such as bars, can increase power within a narrow frequency band, 35-50 Hz (Gray and Singer, 1989). Many other studies, including our own (Cho et al., 2015), have described " $\gamma$ oscillations" corresponding to increased power across a broad frequency range (e.g., $30-100 \mathrm{~Hz}$ ). An open question is whether all of these represent similar phenomena generated by similar mechanisms and mediating similar functions, or whether there are important differences between these various " $\gamma$ oscillations."

$\gamma$ oscillations tend to increase in response to specific task demands. For example, in sensory cortices, attention modulates $\gamma$ oscillations (Fries et al., 2001). During a working memory task, the power of $\gamma$ oscillations in frontal regions increases with the number of items being stored (Roux et al., 2012). One of the most provocative features of $\gamma$ oscillations is that, even though they are generated locally, they can be synchronized across long distances in ways that correlate with behavior. For example, a recent study in monkeys found that $\gamma$ frequency activity recorded from regions of the frontal eye fields and V4 that correspond to the same receptive fields synchronize specifically when attention is directed to that common region of visual space (Gregoriou et al., 2009). $\gamma$ rhythms also synchronize activity across multiple hippocampal subfields and the entorhinal cortex (Colgin et al., 2009; Carr et al., 2012). In this case, synchronization specifically at slow (e.g., $30-50 \mathrm{~Hz}$ ) versus fast $(60-100 \mathrm{~Hz}) \gamma$ frequencies correlates with encoding of future trajectories versus the current location (Zheng et al., 2016). Synchronization between spiking in prefrontal neurons and $\gamma$ oscillations recorded in the hippocampus also appears to be necessary for successful encoding of spatial information during a working memory task (Spellman et al., 2015; Tamura et al., 2016).

\section{Do $\gamma$ oscillations contribute to brain function?}

Together, these observations raise the intriguing possibility that $\gamma$ oscillations play a role in the function of neural circuits. Of course, this need not be the case. Oscillations are general features of circuits that include feedback inhibition, so when interconnected pyramidal neurons and PV interneurons receive sufficient drive, they tend to generate $\gamma$ oscillations (Sohal et al., 2009), and $\gamma$ oscillations may simply reflect these excitatory-inhibitory interactions (Ray et al., 2013). Furthermore, when two regions both generate $\gamma$ oscillations, then the mere presence of connections between them could lead to $\gamma$ frequency synchronization, even when it is of no functional significance. Thus, the real question is as follows: do $\gamma$ oscillations simply reflect levels of local circuit activity, and does long-range $\gamma$-frequency synchronization simply indicate the presence of interactions between two regions? Or, do $\gamma$ oscillations in some way contribute to information processing within local circuits or facilitate communication between different brain regions?

There are many hypothesized mechanisms through which $\gamma$ oscillations may contribute to information processing and/or inter-regional communication. At the same time, numerous studies have observed variability in the frequency of $\gamma$ oscillations, and this had led to appropriate questions about whether these hypothetical mechanisms are robust to such variability (Ray and Maunsell, 2015). In this context, it is important to note that many hypothesized mechanisms involving $\gamma$ oscillations are based on observations that, when $\gamma$ oscillations are present, they have certain effects on information processing within cortical circuits. Many of these effects, described below in more detail, do not depend on a specific oscillation frequency, long-range synchronization, or any additional constraints on circuit wiring. The same way that temporal summation emerges naturally from the gradual decay of synaptic currents, these effects on information processing represent inherent consequences of generic cortical circuit motifs (e.g., convergent patterns of connectivity, feedforward inhibition, etc.). In other words, many studies have shown that, when $\gamma$ oscillations occur, they impact information processing, so arguing that these $\gamma$ oscillation-dependent phenomena do not "contribute" to information processing suggests that, when present, they impact circuit function but do so in haphazard and potentially deleterious ways.

\section{How might $\gamma$ oscillations contribute to circuit function?}

Mechanisms through which $\gamma$ oscillations may contribute to circuit function can be classified based on the circuit locus on which they are hypothesized to act (Fig. 1). Specifically, $\gamma$ oscillations (i.e., $\gamma$-frequency fluctuations in inhibition) may act on local excitatory neurons: (1) to regulate their responses to incoming input, (2) to modulate emergent patterns of local circuit activity, or (3) to enhance the efficacy of output from those neurons onto a downstream target. Although not completely distinct, these categories represent a framework for organizing the diverse mechanisms through which $\gamma$ oscillations may act. Importantly, all of the mechanisms described below assume that information is transmitted by the rate of output spikes (rate coding); they do not rely on phase coding, in which information would be encoded by the particular phase (relative to $\gamma$ ) at which a neuron spikes. Thus, these mechanisms should be distinguished from the hypothesis that $\gamma$ oscillations establish a kind of clock, relative to which the precise timing of spikes carries information (Fries et al., 2007). Similarly, the mechanisms discussed below are all distinct from the proposal of "binding by synchrony" (Singer, 1993), according to which the relative timing of spikes in different neurons carries information about whether those neurons participate in a common neural representation.

Given that $\gamma$ oscillations involve rhythmic fluctuations in levels of inhibition, they should elicit rhythmic changes in neuronal excitability and responsiveness to input. Consistent with this prediction, when PV interneurons are optogenetically stimulated at $40 \mathrm{~Hz}$ (Cardin et al., 2009), then the responses of somatosensory neurons to whisker deflections depend on the phase of the optogenetically induced $\gamma$ oscillation: responses are strongest at phases of $\gamma$ that correspond to the lowest levels of inhibition. The idea that most phases of a $\gamma$ oscillation are dominated by strong inhibition that significantly dampens responses to input, and that the ability to respond to input is maximized during a relatively small window of $\gamma$ phases, is the basis for the communication through coherence (CTC) hypothesis (Fries, 2005, 2015). According to CTC, the flow of information from one structure to another is optimized when $\gamma$ oscillations in these two structures are synchronized so that spikes from the presynaptic structure arrive in the postsynaptic structure at $\gamma$ phases corresponding to this window of maximum responsiveness. CTC requires that oscillations in different brain regions be precisely synchronized, and some studies have found evidence for this. For example, consistent with the idea that phase relationships modulate effectivity connectivity, one study in cat and monkey visual areas found that the phase relationship between activity in two elec- 


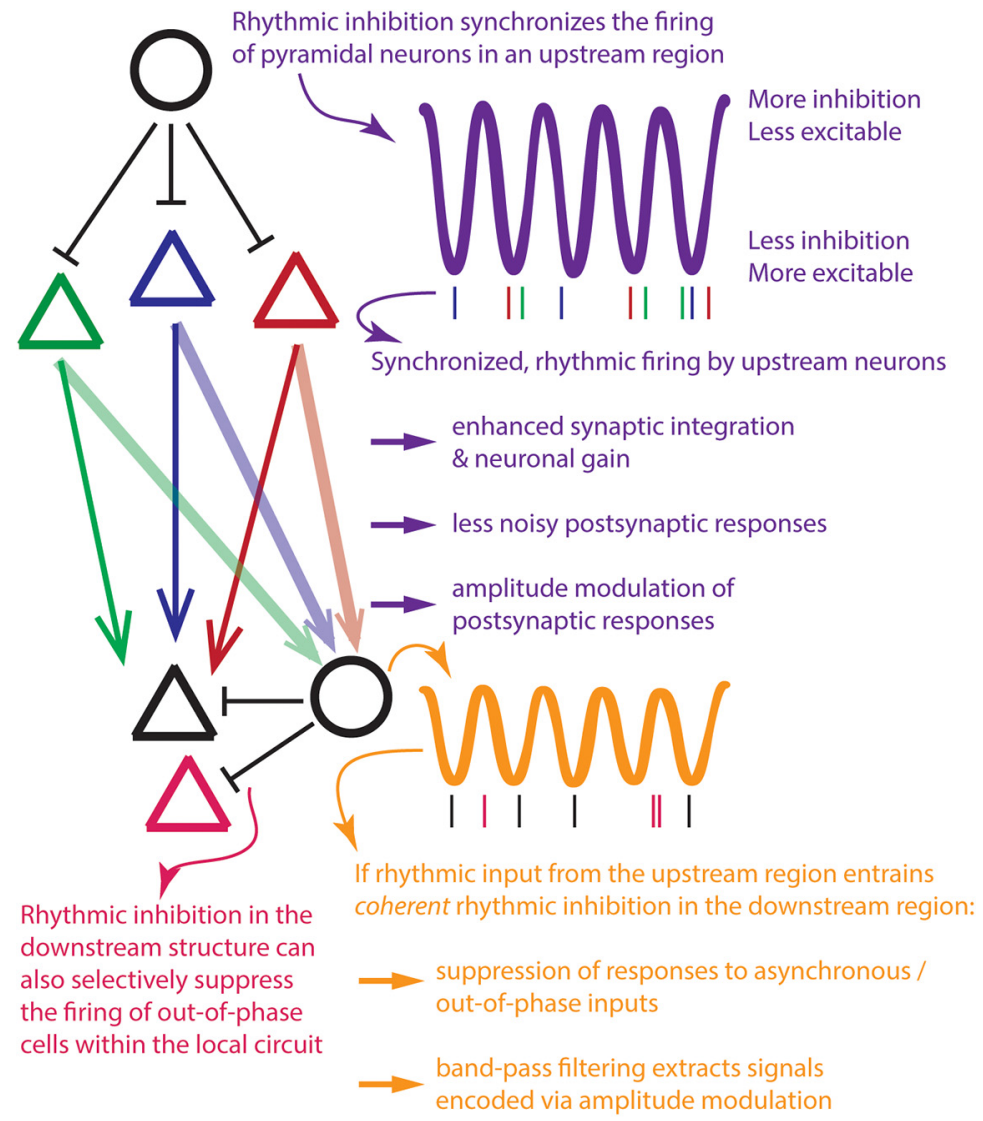

Non-coherent rhythmic inhibition samples different levels of inhibition at different phases of gamma

Figure 1. Schematic overview of mechanisms through which $\gamma$ oscillations might modulate cortical information processing. Top, An inhibitory interneuron (circle) delivers synchronized, rhythmic inhibition to pyramidal neurons (red, blue, and green triangles) in an upstream region, entraining their spiking to the local $\gamma$ rhythm. (Red, blue, and green spikes are concentrated around the troughs of the local $\gamma$ rhythm, represented by the purple waveform to the upper right.) Bottom, The upstream pyramidal neurons send excitatory projections (arrows) to pyramidal neurons (triangles) and an inhibitory interneuron (circle) in a downstream region. Within the downstream region, the inhibitory interneuron (circle) inhibits local pyramidal neurons. Local pyramidal neurons may spike in-phase (black spikes) or out-ofphase (magenta spikes) with $\gamma$ oscillations in the downstream region (represented by the orange waveform to the bottom right), which may or may not be coherent with $\gamma$ oscillations in the upstream region. Mechanisms through which $\gamma$ oscillations may modulate cortical information processing can be divided into three categories based on where within this circuit framework they act. Top right, Purple represents mechanisms through which $\gamma$ oscillations in an upstream region can modulate the flow of information from that region to downstream targets. Bottom right, Orange represents mechanisms through which $\gamma$ oscillations in a downstream region can modulate the responses of that region to incoming input. Some (but not all) of these mechanisms require that $\gamma$ oscillations in the upstream and downstream regions be synchronized or coherent. Bottom left, Magenta represents mechanisms through which $\gamma$ oscillations can shape emergent patterns of local circuit activity.

trodes can predict the correlation between power fluctuations in those two electrodes a few milliseconds later (Womelsdorf et al., 2007). Another study found that, when projections from two sites in V1 converge onto a single target in V4, $\gamma$ oscillations in the target region selectively synchronize with the attended, behaviorally relevant V1 site (Bosman et al., 2012). In the monkey visual cortex, even when the frequency of $\gamma$ oscillations varies as a result of changes in stimulus contrast, this frequency remains matched, and oscillations remain coherent, across V1 and V2 (Roberts et al., 2013). By contrast, other studies have emphasized that $\gamma$ frequencies are highly variable across different visual regions in monkeys (Ray and Maunsell, 2010), and that in cat V1, phase-locking to $\gamma$ is relatively weak (Martin and Schroder, 2016).

The CTC hypothesis has been very influential, inspiring some of the subsequent studies outlined below. However, unlike CTC, many of the mechanisms described below do not require long-distance synchronization between upstream and downstream regions. Rather, they simply require the presence of synchronized rhythmic activity in an upstream region. Of course, these mechanisms are also not mutually exclusive with CTC. Indeed, in many cases, the presence of synchronized rhythmic activity in an upstream region would be expected to entrain inhibition in the downstream region, leading to emergent synchronization that engages CTC, as described below.

One example of a mechanism through which $\gamma$ oscillations in an upstream region could impact information processing in a downstream region is "oscillation-facilitated stimulus selection” (Börgers and Kopell, 2008; Akam and Kullmann, 2010). Suppose that the firing of neurons in an upstream region is synchronized at $\gamma$ frequency. This rhythmic input should rhythmically modulate firing in the downstream structure. As a result, if the input encodes a signal (e.g., a spatial pattern of firing), then that signal will become encoded as a pattern of amplitude modulation of their firing rates of the downstream neurons (Akam and Kullmann, 2010). This pattern of amplitude modulation is robust to other sources of input that are either nonrhythmic or synchronized at other frequencies. In addition, the synchronized nature of the rhythmic input makes it more likely to elicit a response in the downstream target neurons (Börgers and Kopell, 2008). Rhythmic inhibition in the downstream structure is not strictly required for these actions. However, by entraining feedforward inhibition in the target structure, a synchronized $\gamma$ frequency input can also act to suppress responses to competing inputs that are less synchronized or out of phase (Börgers and Kopell, 2008). Indeed, feedforward inhibition can act as a bandpass filter to extract the signal originally encoded by the rhythmic input (Akam and Kullmann, 2010). These latter mechanisms, in which rhythmic input entrains inhibition within the downstream structure, represent important examples of how CTC may work. The mechanisms underlying oscillation-facilitated stimulus selection are also compatible with "oscillatory multiplexing," in which multiple input signals are transmitted using different frequencies (Akam and Kullmann, 2014). Indeed, the ability of the CA1 region of the hippocampus to selectively synchronize with either CA3 at "slow" $\gamma$ frequencies or entorhinal cortex at "fast" $\gamma$ frequencies (Colgin et al., 2009; Carr et al., 2012) may represent evidence for this kind of a mechanism.

In earlier experimental work, we demonstrated a conceptually related phenomenon, in which neurons transmit more information (via their output spike rate) about $\gamma$-frequency inputs than about nonrhythmic ones (Sohal et al., 2009). This "oscillationenhanced rate coding" occurs because the output spike rate elic- 
ited by a given level of input is less noisy in response to rhythmic input than for asynchronous input. Consistent with this idea, a recent experimental study found that noise correlations depend on $\gamma$ phase and power (Womelsdorf et al., 2012). Oscillationenhanced rate coding relies only on the presence of $\gamma$-frequency input (presumably generated by rhythmic inhibition in the upstream structure); neither rhythmic inhibition in the downstream structure nor synchronization between the upstream and downstream regions is required.

$\gamma$ oscillations can also impact rate coding by recruiting physiological mechanisms to modulate synaptic integration and neuronal gain (Salinas and Sejnowski, 2001; Tiesinga et al., 2004; Buzsáki, 2005). In particular, synchronized inputs can increase the probability that a downstream neuron will fire (compared with the asynchronous case) by summating more effectively, engaging nonlinearities involved in spike generation (Azouz and Gray, 2000), and increasing fluctuations in neurons with balanced levels of inhibition and excitation (Tiesinga et al., 2004). These mechanisms are similar to aspects of oscillatory-facilitated stimulus selection and oscillation-enhanced rate coding, in that they depend solely on $\gamma$ oscillations in an upstream region and not on longdistance synchronization. They are also distinct from these mechanisms in important ways. In particular, oscillationenhanced rate coding does not arise from the tendency of $\gamma$-synchronized input to elicit more spikes in a downstream target but rather reflects the tendency of such input to elicit more consistent/less variable levels of spiking in that downstream target (compared with asynchronous input) (Sohal et al., 2009).

All of the mechanisms described above address ways in which $\gamma$ oscillations may affect communication between groups of neurons ( $\gamma$ oscillations in an upstream region can affect its impact on downstream targets, or $\gamma$ oscillations in a downstream region can affect its responses to input). Importantly, all of these mechanisms are also dynamic. As a result, even when a target region receives many sources of input, it can selectively transmit information about a particular one, the identity of which is determined by oscillations, which can be rapidly switched on or off. In addition to these mechanisms, through which $\gamma$ oscillations can flexibly reroute the flow of information between groups of neurons, $\gamma$ oscillations can also modulate patterns of activity within local circuits by increasing or decreasing the firing rates of various neuronal populations. Specifically, a recent study found that, on successful trials of an attention task, a significant fraction of prefrontal pyramidal cells phase-lock to ongoing $\gamma$ oscillations (Kim et al., 2016). $\gamma$ power in the LFP increased during correct trials; and during correct trials, phase-locked neurons that fired near the trough of $\gamma$ (when inhibition should be lowest) increased their firing, whereas those that fired near the peak of $\gamma$ (when inhibition should be strongest) decreased their firing. This suggests that, for the subset of pyramidal neurons that synchronize to $\gamma$ oscillations, increases in $\gamma$ power may concentrate inhibition around particular phases of $\gamma$. This would suppress activity in those cells, that fire at high-inhibition phases and increase activity in those cells that fire at the opposite phases, which are associated with less inhibition. One intriguing possibility is that the neuronal populations that fire at distinct phases of $\gamma$, and consequently increase or decrease their firing in response to $\gamma$ oscillations, represent distinct subtypes of pyramidal neurons, which send output to different targets and thus elicit different effects on behavior (in this scenario, the phase at which an individual neuron fires may be fixed and need not encode any specific information about the stimulus or task). For example, our previous work has shown that, in deep layers of the prefrontal cortex, fast-spiking PV interneurons differentially innervate two subpopulations of pyramidal neurons, which differ in their projection targets, electrophysiological properties, and morphology (Lee et al., 2014b), suggesting that these two subtypes may be differentially modulated by PV interneuron-driven $\gamma$ oscillations. Consistent with this idea, the ability of $\gamma$-frequency inhibition to entrain firing differs across neuronal classes (Otte et al., 2010; Hasenstaub et al., 2016), and studies in monkeys have found cell type-specific synchronization with $\gamma$ oscillations (Gregoriou et al., 2012), and distinct patterns of interlaminar and interregional propagation for $\alpha$ versus $\gamma$ oscillations (van Kerkoerle et al., 2014).

\section{Behavioral effects following manipulations of $\boldsymbol{\gamma}$ oscillations}

The preceding suggests that there are multiple mechanisms through which $\gamma$ oscillations could impact circuit function, and ultimately, behavior. Recently, three studies have found evidence for behavioral effects following optogenetic manipulations of $\gamma$ oscillations. The first of these (Siegle et al., 2014) showed that 40 $\mathrm{Hz}$ optogenetic stimulation of PV interneurons in the somatosensory cortex could enhance the detection of "less salient" tactile stimuli (i.e., those stimuli that were most difficult to detect at baseline). Notably, this $\gamma$-frequency optogenetic stimulation actually interfered with the detection of "more salient" stimuli (i.e., the ones that were easiest to detect at baseline). To determine whether the enhanced detection of less salient stimuli depends specifically on the induction of $\gamma$ rhythms, this study then looked at the detection of stimuli that were only presented at specific phases relative to the optogenetically entrained $\gamma$ rhythm. If the effects of optogenetically stimulating PV interneurons on less salient stimuli simply reflect a temporally nonspecific increase in the level of inhibition, then the enhanced detection should occur regardless of this phase relationship. However, the authors observed that detection was selectively enhanced when sensory input arrived in the cortex just before a bout of PV interneurondriven inhibition. This was consistent with earlier work from the same group, which showed that both the responsiveness to sensory input and temporal precision of such responses was greatest at this phase of an optogenetically induced $\gamma$ oscillation, which should be associated with the lowest levels of inhibition (Cardin et al., 2009). Thus, the authors hypothesized that sensory inputs arriving during this window would be more reliably transmitted to downstream structures, leading to the improved behavioral detection they had observed. The authors further hypothesized that, even during non-phase-locked naturalistic sensory stimulation, high-velocity micromotions occurring several times per $\gamma$ cycle might lead to sampling of the stimulus at optimal phases of $\gamma$.

A study from our laboratory focused on the role of prefrontal $\gamma$ oscillations in cognitive flexibility (Cho et al., 2015). We studied mutant mice in which abnormal prefrontal PV interneuron physiology, markedly impaired cognitive flexibility, and deficient taskevoked $\gamma$ oscillations all appear after adolescence, modeling aspects of schizophrenia. We expressed ChR2 selectively within prefrontal interneurons and found that 40 or $60 \mathrm{~Hz}$ optogenetic stimulation of prefrontal interneurons completed normalized cognitive flexibility in these mutant mice. Furthermore, this rescue was frequencyspecific and persistent: stimulating prefrontal interneurons using a combination of higher and lower frequencies (12.5 and $125 \mathrm{~Hz}$ ) failed to even partially rescue cognitive flexibility in mutant mice, whereas mice that received $\gamma$-frequency stimulation during one task 
day were able to perform normally up to 1 week later in the absence of additional stimulation. These results demonstrate how interneuron-driven $\gamma$ oscillations in the prefrontal cortex can potently regulate cognitive flexibility, although the exact mechanism through which this occurs remains unclear.

Another study has explored the effect of stimulating prefrontal PV interneurons during an attentional task in which mice must use a brief visual cue to guide a nose poke into one of three possible locations (Kim et al., 2016). This study found that correct performance is associated with a robust increase in PV interneuron activity and increased $\gamma$ synchronization of both PV neurons and a subset of presumed pyramidal neurons. Furthermore, optogenetic stimulation of PV interneurons at $30-40 \mathrm{~Hz}$ improved performance, whereas stimulation at $1-10 \mathrm{~Hz}$ produced a much larger decrement in performance. The authors hypothesized that $\gamma$-frequency $(30-40 \mathrm{~Hz})$ stimulation may improve performance by synchronizing pyramidal neuron firing. Furthermore, as mentioned earlier, this study found that increases in $\gamma$ oscillations are associated with enhanced and suppressed firing of pyramidal neurons that phase-lock to the troughs or peaks of $\gamma$, respectively.

\section{Caveats and considerations about optogenetic manipulations of $\gamma$ oscillations}

All three studies outlined above performed important controls, which suggest that the behavioral improvements associated with $\gamma$-frequency stimulation of interneurons depend specifically on the induction of $\gamma$ rhythms and are not simply consequences of increased overall levels of inhibition. In Siegle et al. (2014), improvements in the detection of less salient tactile stimuli only occur at certain phases relative to the optogenetically induced $\gamma$ rhythm. In Cho et al. (2015), the effects of $\gamma$ frequency stimulation are not reproduced, even partially, by delivering an equal amount of light stimulation at a combination of higher and lower frequencies. In Kim et al. (2016), the effects of stimulation at lower frequencies go in the opposite direction compared with $\gamma$ frequency stimulation. Of course, these controls are not bulletproof. In the case of Siegle et al. (2014), one could argue that various changes in steady-state levels of inhibition (either increases or decreases) might reproduce the range of observed behavioral effects. Thus, the distinct behavioral effects (improved vs worsened detection) observed at different $\gamma$ phases could simply reflect the fact that different phases correspond to different levels of inhibition. Thus, the same effects may have been obtained by appropriately tuning the level of tonic inhibition (although even in this scenario, rhythmicity represents a useful mechanism for sampling various levels of inhibition when the appropriate one is not known a priori). For Cho et al. (2015), one could argue that delivering equal amounts of light stimulation fails to recruit PV interneurons as effectively as $\gamma$ frequency stimulation, and that increasing inhibition has an "all or none effect," such that $\gamma$ frequency stimulation always crosses some threshold for the level of inhibition required to normalize behavior, whereas $12.5 \mathrm{~Hz} / 125$ $\mathrm{Hz}$ stimulation never crosses this threshold. In the case of Kim et al. (2016), lower frequencies of stimulation will elicit less inhibition, and one could argue that inhibition has a biphasic effect whereby lower levels of inhibition elicit qualitatively different effects than higher levels of inhibition. Occum's razor would seem to make all of these hypothesized scenarios less likely than the authors' favored interpretations, although that does not rule them out entirely.

A more fundamental issue with criticisms like these, which argue that manipulations of $\gamma$ oscillations actually end up manip- ulating "something else" (e.g., levels of inhibition) and that this "something else" is indeed the key driver of behavioral effects, is that they cannot easily be falsified. That is to say, no matter how carefully these manipulations and control experiments are designed, there will always be "something else" that also ends up being manipulated and could plausibly contribute to observed behavioral effects. The reason is that $\gamma$ oscillations, by their nature, involve excitatory and inhibitory synaptic interactions that modulate neuronal firing. Thus, there is simply no way to manipulate $\gamma$ oscillations without manipulating numerous other aspects of circuit activity. This issue is not unique to $\gamma$ oscillations. Electrical microstimulation and optogenetic stimulation, which often purport to selectively increase or decrease spiking within one brain region or cell type, invariably elicit numerous unintended effects (e.g., altered activity in interconnected cell types and brain regions) (Otchy et al., 2015). Indeed, any manipulation that increases the firing of cortical excitatory neurons is almost certain to also increase $\gamma$ oscillations. Yet the conventional interpretation of such experiments is to attribute observed behavioral effects to the directly stimulated neuronal firing, rather than indirect modulations of $\gamma$ oscillations or other off-target effects.

\section{Where do we go from here?}

What is generally agreed upon is that $\gamma$ oscillations occur frequently in active cortical circuits, where they reflect interactions between excitatory and inhibitory neurons. However, this does not mean that $\gamma$ oscillations are simply generic consequences of such interactions. Rather, the intrinsic and synaptic properties of fast-spiking PV interneurons seem exquisitely tuned to produce $\gamma$ oscillations that are synchronized at specific frequencies (Galarreta and Hestrin, 1999; Gibson et al., 1999; Tamás et al., 2000; Bartos et al., 2002). Furthermore, comparing the properties of fast-spiking PV interneurons with those of other interneuron populations makes clear that this need not have been the case. Thus, if $\gamma$ oscillations are simply byproducts of excitatoryinhibitory interactions, the same way that the sound of an engine is a byproduct of internal combustion, then $\gamma$ oscillations seem to most closely resemble the roar of an Italian sports car, which may have originated as an epiphenomenon, but has since been purposefully calibrated to achieve a desired effect.

As outlined above, when $\gamma$ oscillations occur, they often synchronize at least a subset of pyramidal neurons, and this alone is enough to have important consequences for rate coding, regardless of the precise oscillation frequency or the presence of longrange synchronization. Of course, long-range synchronization is frequently observed, and recent studies have uncovered fastspiking PV-expressing GABAergic neurons that project longdistances (e.g., across the corpus callosum or from the cortex or to subcortical targets) (Caputi et al., 2013; Lee et al., 2014a). These long-range projecting, fast-spiking PV neurons may serve to facilitate long-range $\gamma$ synchronization.

Thus, the key challenge moving forward is to more directly link specific physiological mechanisms (e.g., communication through coherence, stimulus selection, oscillatory multiplexing, oscillationenhanced rate coding, etc.) with the behavioral effects that appear to follow acute manipulations of $\gamma$ oscillations. Future studies should demonstrate that these manipulations elicit specific physiological correlates of these mechanisms. For example, does optogenetically induced $\gamma$-frequency synchronization in an upstream region elicit phase-locked inhibition and/or coherent $\gamma$ oscillations in a downstream target? Can we control the relative contributions of multiple inputs to a common target by manipulating $\gamma$-frequency synchronization within each upstream region? Does the induction of $\gamma$ oscil- 
lations in a local circuit reshape its pattern of output? Elucidating which mechanisms $\gamma$ oscillations actually use to influence behavior is not merely an academic exercise, as disruptions in interneuron generated $\gamma$ oscillations are hallmarks of neuropsychiatric disorders, such as schizophrenia (Gonzalez-Burgos et al., 2015; Senkowski and Gallinat, 2015). Thus, if $\gamma$ oscillations do indeed contribute to circuit function, then interventions that normalize the relevant mechanisms may represent powerful new therapies for the currently intractable cognitive deficits at the core of these disorders (Cho et al., 2015).

\section{Response from Dual Perspective Companion Author- Jessica Cardin}

Vikaas' perspective piece provides an insightful and balanced view of the functional role of $\gamma$ oscillations. He makes a strong case that $\gamma$ oscillations affect information processing in cortical circuits, and highlights several key mechanisms by which that regulation might be effected.

Importantly, Vikaas' piece highlights not only the influential communication through coherence hypothesis, but also several other categories of mechanism by which $\gamma$ oscillations may regulate information encoding and transmission. These include oscillation-facilitated stimulus selection (Börgers et al., 2008; Akam and Kullmann, 2010), oscillation-enhanced rate coding (Sohal et al., 2009), and modulation of synaptic integration and gain control. Whereas communication through coherence requires long-distance synchronization, the other mechanisms do not, indicating a variety of potential ways for local $\gamma$ oscillations to influence information encoding and transmission. The functional roles of $\gamma$ may thus be as diverse as the cellular and circuit mechanisms by which these oscillations are generated in the cortex and other structures.

Vikaas further points out the tremendous complexity of directly testing the contribution of any of the possible mechanisms by which $\gamma$ might regulate information processing. One of the key caveats to any manipulation is that, as stated in Vikaas' review, one cannot manipulate $\gamma$ without altering other aspects of local or long-range circuit activity. Several groups have begun to test the functional role of $\gamma$ oscillations by optogenetically manipulating PV interneurons (Siegle et al., 2014; Cho et al., 2015; Kim et al., 2016). However, current evidence remains incomplete, and further experimental and computational work is needed. In addition to examining PV interneurons, targeted manipulations of other GABAergic interneurons and specific excitatory neurons may shed light on the inhibitory synaptic interactions that regulate the expression of different forms of $\gamma$ oscillations.

Last, Vikaas makes an important point about the potential translational impact of enhancing our understanding of $\gamma$ generation and function. Dysregulated $\gamma$ activity has been observed in a number of human psychiatric disorders and remains a key hallmark that can be usefully examined in animal models.

\section{References}

Akam T, Kullmann DM (2010) Oscillations and filtering networks support flexible routing of information. Neuron 67: 308-320. CrossRef Medline

Börgers C, Epstein S, Kopell NJ (2008) $\gamma$ oscillations mediate stimulus competition and attentional selection in a cortical network model. Proc Natl Acad Sci U S A 105:18023-18028. CrossRef Medline

Cho KK, Hoch R, Lee AT, Patel T, Rubenstein JL, Sohal VS (2015) $\gamma$ rhythms link prefrontal interneuron dysfunction with cognitive inflexibility in Dlx5/6(+/-) mice. Neuron 85:1332-1343. CrossRef Medline

Kim H, Ährlund-Richter S, Wang X, Deisseroth K, Carlén M (2016) Prefrontal parvalbumin neurons in control of attention. Cell 164:208-218. CrossRef Medline

Siegle JH, Pritchett DL, Moore CI (2014) $\gamma$-range synchronization of fast-spiking interneurons can enhance detection of tactile stimuli. Nat Neurosci 17:1371-1379. CrossRef Medline

Sohal VS, Zhang F, Yizhar O, Deisseroth K (2009) Parvalbumin neurons and $\gamma$ rhythms enhance cortical circuit performance. Nature 459:698-702. CrossRef Medline

\section{References}

Akam T, Kullmann DM (2010) Oscillations and filtering networks support flexible routing of information. Neuron 67:308-320. CrossRef Medline

Akam T, Kullmann DM (2014) Oscillatory multiplexing of population codes for selective communication in the mammalian brain. Nat Rev Neurosci 15:111-122. CrossRef Medline

Azouz R, Gray CM (2000) Dynamic spike threshold reveals a mechanism for synaptic coincidence detection in cortical neurons in vivo. Proc Natl Acad Sci U S A 97:8110-8115. CrossRef Medline

Bartos M, Vida I, Frotscher M, Meyer A, Monyer H, Geiger JR, Jonas P (2002) Fast synaptic inhibition promotes synchronized $\gamma$ oscillations in hippocampal interneuron networks. Proc Natl Acad Sci U S A 99:1322213227. CrossRef Medline

Bartos M, Vida I, Jonas P (2007) Synaptic mechanisms of synchronized $\gamma$ oscillations in inhibitory interneuron networks. Nat Rev Neurosci 8:4556. CrossRef Medline

Börgers C, Kopell NJ (2008) $\gamma$ oscillations and stimulus selection. Neural Comput 20:383-414. CrossRef Medline

Bosman CA, Schoffelen JM, Brunet N, Oostenveld R, Bastos AM, Womelsdorf T, Rubehn B, Stieglitz T, De Weerd P, Fries P (2012) Attentional stimulus selection through selective synchronization between monkey visual areas. Neuron 75:875-888. CrossRef Medline

Buzsáki G (2005) Rhythms of the brain. Oxford: Oxford UP.

Buzsáki G, Wang XJ (2012) Mechanisms of $\gamma$ oscillations. Annu Rev Neurosci 35:203-225. CrossRef Medline

Caputi A, Melzer S, Michael M, Monyer H (2013) The long and short of GABAergic neurons. Curr Opin Neurobiol 23:179-186. CrossRef Medline

Cardin JA, Carlén M, Meletis K, Knoblich U, Zhang F, Deisseroth K, Tsai LH, Moore CI (2009) Driving fast-spiking cells induces $\gamma$ rhythm and controls sensory responses. Nature 459:663-667. CrossRef Medline

Carr MF, Karlsson MP, Frank LM (2012) Transient slow $\gamma$ synchrony underlies hippocampal memory replay. Neuron 75:700-713. CrossRef Medline

Cho KK, Hoch R, Lee AT, Patel T, Rubenstein JL, Sohal VS (2015) $\gamma$ rhythms link prefrontal interneuron dysfunction with cognitive inflexibility in Dlx5/6(+/-) mice. Neuron 85:1332-1343. CrossRef Medline

Colgin LL, Denninger T, Fyhn M, Hafting T, Bonnevie T, Jensen O, Moser MB, Moser EI (2009) Frequency of $\gamma$ oscillations routes flow of information in the hippocampus. Nature 462:353-357. CrossRef Medline

Csicsvari J, Jamieson B, Wise KD, Buzsáki G (2003) Mechanisms of $\gamma$ oscillations in the hippocampus of the behaving rat. Neuron 37:311-322. CrossRef Medline

Fries P (2005) A mechanism for cognitive dynamics: neuronal communication through neuronal coherence. Trends Cogn Sci 9:474-480. CrossRef Medline

Fries P (2015) Rhythms for cognition: communication through coherence. Neuron 88:220-235. 
Fries P, Reynolds JH, Rorie AE, Desimone R (2001) Modulation of oscillatory neuronal synchronization by selective visual attention. Science 291: 1560-1563. CrossRef Medline

Fries P, Nikolić D, Singer W (2007) The $\gamma$ cycle. Trends Neurosci 30:309316. CrossRef Medline

Galarreta M, Hestrin S (1999) A network of fast-spiking cells in the neocortex connected by electrical synapses. Nature 402:72-75. CrossRef Medline

Gibson JR, Beierlein M, Connors BW (1999) Two networks of electrically coupled inhibitory neurons in neocortex. Nature 402:75-79. CrossRef Medline

Gonzalez-Burgos G, Cho RY, Lewis DA (2015) Alterations in cortical network oscillations and parvalbumin neurons in schizophrenia. Biol Psychiatry 77:1031-1040. CrossRef Medline

Gray CM, Singer W (1989) Stimulus-specific neuronal oscillations in orientation columns of cat visual cortex. Proc Natl Acad Sci U S A 86:16981702. CrossRef Medline

Gregoriou GG, Gotts SJ, Zhou H, Desimone R (2009) Long-range neural coupling through synchronization with attention. Prog Brain Res 176:3545. CrossRef Medline

Gregoriou GG, Gotts SJ, Desimone R (2012) Cell-type-specific synchronization of neural activity in FEF with V4 during attention. Neuron 73:581594. CrossRef Medline

Hasenstaub A, Shu Y, Haider B, Kraushaar U, Duque A, McCormick DA (2005) Inhibitory postsynaptic potentials carry synchronized frequency information in active cortical networks. Neuron 47:423-435. CrossRef Medline

Hasenstaub A, Otte S, Callaway E (2016) Cell type-specific control of spike timing by $\gamma$-band oscillatory inhibition. Cereb Cortex 26:797-806. CrossRef Medline

Kim H, Ährlund-Richter S, Wang X, Deisseroth K, Carlén M (2016) Prefrontal parvalbumin neurons in control of attention. Cell 164:208-218. CrossRef Medline

Lee AT, Vogt D, Rubenstein JL, Sohal VS (2014a) A class of GABAergic neurons in the prefrontal cortex sends long-range projections to the nucleus accumbens and elicits acute avoidance behavior. J Neurosci 34: 11519-11525. CrossRef Medline

Lee AT, Gee SM, Vogt D, Patel T, Rubenstein JL, Sohal VS (2014b) Pyramidal neurons in prefrontal cortex receive subtype-specific forms of excitation and inhibition. Neuron 81:61-68. CrossRef Medline

Martin KA, Schroder S (2016) Phase locking of multiple single neurons to the local field potential in Cat V1. J Neurosci 36:2494-2502.

Otchy TM, Wolff SB, Rhee JY, Pehlevan C, Kawai R, Kempf A, Gobes SM, Ölveczky BP (2015) Acute off-target effects of neural circuit manipulations. Nature 528:358-363. CrossRef Medline

Otte S, Hasenstaub A, Callaway EM (2010) Cell type-specific control of neuronal responsiveness by $\gamma$-band oscillatory inhibition. J Neurosci 30: 2150-2159. CrossRef Medline

Ray S, Maunsell JH (2010) Differences in $\gamma$ frequencies across visual cortex restrict their possible use in computation. Neuron 67:885-896. CrossRef Medline

Ray S, Maunsell JH (2015) Do $\gamma$ oscillations play a role in cerebral cortex? Trends Cogn Sci 19:78-85. CrossRef Medline

Ray S, Ni AM, Maunsell JH (2013) Strength of $\gamma$ rhythm depends on normalization. PLoS Biol 11:e1001477. CrossRef Medline

Roberts MJ, Lowet E, Brunet NM, Ter Wal M, Tiesinga P, Fries P, De Weerd
P (2013) Robust $\gamma$ coherence between macaque V1 and V2 by dynamic frequency matching. Neuron 78:523-536. CrossRef Medline

Roux F, Wibral M, Mohr HM, Singer W, Uhlhaas PJ (2012) $\gamma$-band activity in human prefrontal cortex codes for the number of relevant items maintained in working memory. J Neurosci 32:12411-12420. CrossRef Medline

Salinas E, Sejnowski TJ (2001) Correlated neuronal activity and the flow of neural information. Nat Rev Neurosci 2:539-550. CrossRef Medline

Senkowski D, Gallinat J (2015) Dysfunctional prefrontal $\gamma$-band oscillations reflect working memory and other cognitive deficits in schizophrenia. Biol Psychiatry 77:1010-1019. CrossRef Medline

Siegle JH, Pritchett DL, Moore CI (2014) $\gamma$-range synchronization of fastspiking interneurons can enhance detection of tactile stimuli. Nat Neurosci 17:1371-1379. CrossRef Medline

Singer W (1993) Synchronization of cortical activity and its putative role in information processing and learning. Annu Rev Physiol 55:349-374. CrossRef Medline

Sohal VS, Zhang F, Yizhar O, Deisseroth K (2009) Parvalbumin neurons and $\gamma$ rhythms enhance cortical circuit performance. Nature 459:698702. CrossRef Medline

Spellman T, Rigotti M, Ahmari SE, Fusi S, Gogos JA, Gordon JA (2015) Hippocampal-prefrontal input supports spatial encoding in working memory. Nature 522:309-314.

Tamás G, Buhl EH, Lörincz A, Somogyi P (2000) Proximally targeted GABAergic synapses and gap junctions synchronize cortical interneurons. Nat Neurosci 3:366-371. CrossRef Medline

Tamura M, Mukai J, Gordon JA, Gogos JA (2016) Developmental inhibition of Gsk3 rescues behavioral and neurophysiological deficits in a mouse model of schizophrenia predisposition. Neuron 89:1100-1109.

Tiesinga P, Sejnowski TJ (2009) Cortical enlightenment: are attentional $\gamma$ oscillations driven by ING or PING? Neuron 63:727-732. CrossRef Medline

Tiesinga PH, Fellous JM, Salinas E, José JV, Sejnowski TJ (2004) Inhibitory synchrony as a mechanism for attentional gain modulation. J Physiol Paris 98:296-314. CrossRef Medline

van Kerkoerle T, Self MW, Dagnino B, Gariel-Mathis MA, Poort J, van der Togt C, Roelfsema PR (2014) $\alpha$ and $\gamma$ oscillations characterize feedback and feedforward processing in monkey visual cortex. Proc Natl Acad Sci U S A 111:14332-14341. CrossRef Medline

Whittington MA, Traub RD, Jefferys JG (1995) Synchronized oscillations in interneuron networks driven by metabotropic glutamate receptor activation. Nature 373:612-615. CrossRef Medline

Womelsdorf T, Schoffelen JM, Oostenveld R, Singer W, Desimone R, Engel AK, Fries P (2007) Modulation of neuronal interactions through neuronal synchronization. Science 316:1609-1612. CrossRef Medline

Womelsdorf T, Lima B, Vinck M, Oostenveld R, Singer W, Neuenschwander S, Fries P (2012) Orientation selectivity and noise correlation in awake monkey area V1 are modulated by the $\gamma$ cycle. Proc Natl Acad Sci U S A 109:4302-4307. CrossRef Medline

Yamamoto J, Suh J, Takeuchi D, Tonegawa S (2014) Successful execution of working memory linked to synchronized high-frequency gamma oscillations. Cell 157:845-857.

Zheng C, Bieri KW, Hsiao YT, Colgin LL (2016) Spatial sequence coding differs during slow and fast gamma rhythms in the hippocampus. Neuron 89:398-408. 